\title{
Reactivity of Poly(ethylene oxide) Macromonomers in Radical Copolymerization
}

\author{
Koichi ITo, Hiroyuki Tsuchida, Akio HaYASHI, ${ }^{\dagger}$ \\ Toshiaki KITANO, Eiichi YAMADA,* \\ and Takeo MATSUMOTO* \\ School of Materials Science, Toyohashi University of Technology, \\ Tempaku-cho, Toyohashi 440, Japan \\ *Tsukuba Laboratory, Nippon Oil \& Fats Co., Ltd., Tokodai 5-10, \\ Toyosato-machi, Tsukuba, Ibaraki 300-26, Japan
}

(Received September 26, 1984)

\begin{abstract}
Poly(ethylene oxide) macromonomers carrying $p$-vinylbenyl and methacrylate end-groups were prepared by the anionic polymerization of ethylene oxide followed by reactions with $p$-vinylbenzyl chloride and methacryloyl chloride, respectively. Radical copolymerization with styrene $\left(\mathrm{M}_{1}\right)$ in tetrahydrofuran at $60^{\circ} \mathrm{C}$ was carried out to give the macromonomer's relative reactivity $\left(1 / r_{1}\right)$, which decreased by a factor as much as about $1 / 2$ with increasing degree of polymerization to about 100 . This is discussed in terms of the kinetic excluded volume effect but by taking into consideration the generally repulsive interaction between different polymer chains and/or some different solution behavior of the polymers. These should be important factors in copolymerization involving the macromonomer.
\end{abstract}

KEY WORDS Macromonomer / Poly(ethylene oxide) / Monomer Reactivity Ratio / Polymer Effect on Reactivity / Kinetic Excluded Volume Effect / Polymer-Polymer Interaction /

Recent developments in macromonomer ('macromer') techniques have made possible the preparation of a variety of graft copolymers of well-defined structure, as reviewed by Yamashita ${ }^{1}$ and Rempp and Franta. ${ }^{2}$ However, the relevant copolymerization parameters, which are very important in determining the trunk-branch distribution in the resulting graft copolymers, have been reported in rather limited cases, possibly because of experimental difficulty arising from the high molecular weight itself of the macromonomer compared to the conventional comonomer. Thus in general, particular care should be taken for the accurate determination of the composition and purification of the product free from unreacted macromonomers.

Among available reactivity data, we reported earlier that the methacrylate-ended poly(methyl methacrylate) macromonomer copolymerized azeotropically with stearyl methacrylate, i.e., $r_{1} \cong r_{2} \cong 1$ in benzene. ${ }^{3}$ On the other hand, the polystyrene macromonomer with a methacrylate end-group showed a significantly lower reactivity, compared to its model monomer, methyl or stearyl methacrylate, in copolymerization with 2-hydroxyethyl methacrylate in dimethylformamide. $^{4-6}$ Schulz and Milkovich ${ }^{7}$ recently presented their data on the copolymerization of the methacrylate-terminated polystyrene macromonomer which showed a similar or a

${ }^{\dagger}$ Present Address: Tsukuba Laboratory, Nippon Oil \& Fats Co., Ltd., Tokodai 5-10, Toyosato-cho, Tsukuba, Ibaraki 300-26, Japan. 

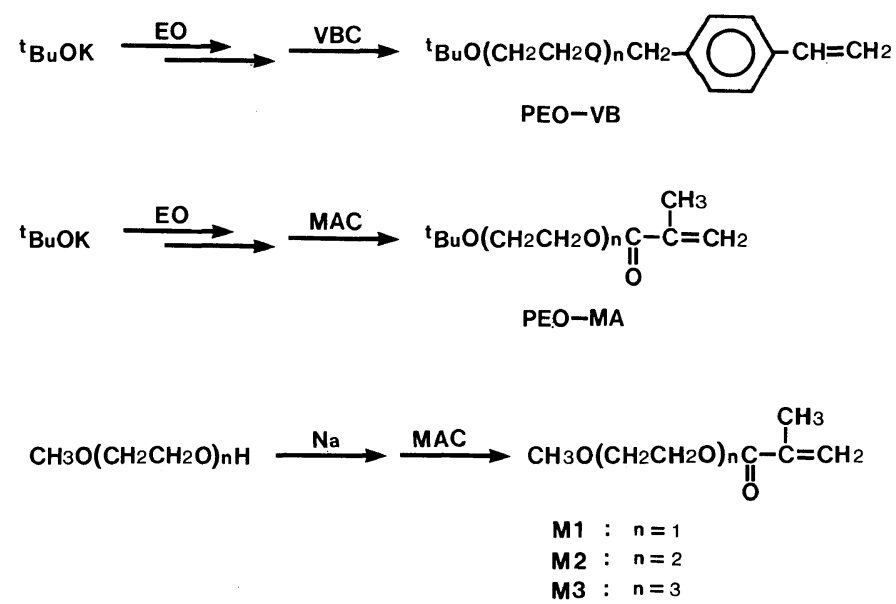

Scheme 1. Preparation of PEO macromonomers and oligomer models.

slightly lower reactivity, depending on the medium, compared to low molecular weight homologs. Similar results have been reported by Asami et $a .^{8}$ with $p$-vinylbenzyl-ended polystyrene or methacrylate- and acrylateended polytetrahydrofuran, and by Kennedy and $\mathrm{Hiza}^{9}$ with methacrylate-ended polyisobutylene macromonomers.

In general, however, more detailed and more systematic investigations are clearly required to understand the macromonomer's reactivity in greater detail. In this paper, we prepared poly(ethylene oxide) (PEO) macromonomers having $p$-vinylbenzyl and methacrylate terminals, according to Scheme 1 , and examined their copolymerization with styrene as a function of molecular weight. The results are discussed in relation to problems encountered in determining the copolymerization parameters and effects of molecular weight on related polymer-polymer reactions. PEO macromonomers have been prepared and are expected to be useful for preparing amphiphilic graft copolymers for a variety of applications. ${ }^{2,10,11}$

\section{EXPERIMENTAL}

\section{Materials}

Commercial reagents were purified accord- ing to usual procedures. Styrene (St) and oligo(ethylene glycol) monomethyl ethers were distilled over calcium hydride under reduced pressure of argon. Tetrahydrofuran (THF) was refluxed and distilled over sodium wire. $\alpha, \alpha^{\prime}$-Azobisisobutyronitrile (AIBN) was recrystallized from methanol. The reagents used for anionic polymerization of ethylene oxide (EO) and its end-capping were purified and dried by the usual procedure using a vacuum line $\left(10^{-5} \mathrm{mmHg}\right)$, and sealed in ampoules fitted with breakable seals. EO was purified by repeated trap-to-trap distillations over calcium hydride and finally distilled over sodium mirror or sodium naphthalene in THF. THF was redistilled from a dark blue solution of sodium anthracene and finally from a red solution of disodium salt of $\alpha$-methylstyrene tetramer. Potassium tert-butoxide $\left(\mathrm{KO}^{t} \mathrm{Bu}\right)$, as a solution in THF, was prepared and purified as described before. ${ }^{12} p$-Vinylbenzyl chloride (VBC) and methacryloyl chloride (MAC) were distlled over calcium hydride and stocked as THF solutions for end-capping.

\section{Macromonomers}

PEO macromonomers with $p$-vinylbenzyl and methacrylate end-groups were prepared by a conventional high vacuum line teehnique. Polymerization of $\mathrm{EO}$ by $\mathrm{KO}^{t} \mathrm{Bu}$ in $\mathrm{THF}$ was 
conducted at $40^{\circ} \mathrm{C}$ overnight to a quantitative conversion to a polymer of the desired degree of polymerization. The resulting PEO potassium alkoxide was subjected to the reaction with more than five-fold excess VBC or MAC for about $1 \mathrm{~h}$. Potassium chloride, precipitated by pouring the reaction mixture into acetone, was filtered off, and the filtrate was evaporated under vacuum to almost dryness and poured into petroleum ether, and finally freeze-dried from benzene. PEO-VB and PEO-MA thus obtained were satisfactorily characterized by VPO, ${ }^{1} \mathrm{H}$ NMR, GPC, and UV, and the results are given in Table I. The numbers following PEO-VB and PEO-MA indicate the numberaverage degree of polymerization of $\mathrm{EO}$ as determined by VPO.

The low molecular weight homologs of PEO-MA, M1, M2, and M3, were prepared by a reaction of the corresponding oligo(ethylene glycol) monomethyl ether with excess sodium in benzene and then with excess MAC, followed by distillation under vacuum: bp 73$75^{\circ} \mathrm{C} / 19 \mathrm{mmHg}$. $\quad$ lit $^{23)} \quad 75-76^{\circ} \mathrm{C} / 22 \mathrm{mmHg}$ (M1), $56-58^{\circ} \mathrm{C} / 1 \mathrm{mmHg}$ (M2), ca. $120^{\circ} \mathrm{C} /$ $1 \mathrm{mmHg}$ (M3). The homologs were confirmed by ${ }^{1} \mathrm{H}$ NMR and gas chromatography to be practically pure.

\section{Copolymerization}

The monomers were weighed into a glass tube, together with AIBN as the initiator and THF as the solvent, and degassed by freezethaw cycles. The tube was then sealed under vacuum and placed in an oil bath of $60^{\circ} \mathrm{C}$ for polymerization. The feed molar ratio of $\mathrm{St}$ to the macromonomer was taken to be sufficiently large (usually greater than approximately 10) to satisfy simplified treatment of copolymerization as will be shown later. It should be noted in this connection that the products rich in St wt \% could be precipitated out of methanol, a nonsolvent for poly-St but a good solvent for the unreacted PEO macromonomer. The products were isolated by a centrifuge (4000 rpm), although considerably stable emulsions in methanol sometimes resulted. Copolymers from PEO-VB-13 and -29 (Tables III and IV) were isolated by precipitation into petroleum ether, followed by repeated washing with water. Almost all the products were checked by GPC and found in every case to be free of the unreacted macromonomers.

Copolymerizations of styrene with M1, M2, and M3 were conducted by the same procedure but over the usual wide range of composition. The copolymers were precipitated and isolated from petroleum ether.

\section{Characterization Methods}

Vapor pressure osmometry (VPO) was measured in benzene at $45^{\circ} \mathrm{C}$ with a Molecular Weight Apparatus of Corona Electric Co., Ltd., Type 117, calibrated with benzyl or standard polystyrenes. UV spectra were recorded on a double-beam spectrophotometer, Hitachi 330, with a monomer solution in THF of spectrum grade. The $p$-vinylbenzyl and methacrylate end-groups showed absorption maxima at 252 and $209 \mathrm{~nm}$, respectively, just as the corresponding polytetrahydrofuran macromonomers reported by Asami et al. ${ }^{13,14}$ The number-average molecular weights of the macromonomers were calculated assuming one double bond per chain with the reported values for the absorption coefficients, $\varepsilon_{\max }=$ $1.59 \times 10^{4}$ and $8.1 \times 10^{3}$, respectively. The absorbance at $209 \mathrm{~nm}$ was, however, not very much reproducible, even under a nitrogen atmosphere, with a relative error of about $\pm 10 \%$.

Gel permeation chromatography (GPC) was taken on a JASCO HPLC, TRIROTAR-III, equipped with RI (Shodex SE-11) and UV (UVIDEC-100-III) detectors, with a column, Shodex A-801 or A-803, calibrated with standard polystyrenes (Polymer Lab., Ltd.) and poly(ethylene oxides) (Toyo Soda Co., Ltd.). Solutions $(0.5-1 \cdot \mathrm{wt} \%)$ were eluted by THF at a flow rate of $1 \mathrm{ml} / \mathrm{min}$ at $40^{\circ} \mathrm{C}$. The molecular weights of some macromonomers were calcu- 
lated from those of polystyrene calibration using the equation proposed by Mori ${ }^{15}$

$$
M_{n, \mathrm{PEO}}=1.21 M_{n, \mathrm{PSt}}^{0.916}
$$

${ }^{1} \mathrm{H}$ NMR spectra were recorded on a JEOL JNM PMX-60 or FX-90 FT spectrometer, with about $5-10 \mathrm{wt} \%$ polymer solutions in $\mathrm{CDCl}_{3}$. The spectra of macromonomers were satisfactory with assignments as follow.

PEO-VB: phenylene $-\mathrm{C}_{6} \mathrm{H}_{4}-(\delta 7.35$; deformed $\mathrm{AB}$ quartet; $4 \mathrm{H}$ ), double bond $-\mathrm{CH}=$ $\left(\delta 6.65, J_{\mathrm{AX}}=11.0, J_{\mathrm{BX}}=17.4 \mathrm{~Hz} ; \mathrm{X}\right.$ part of $\mathrm{ABX} ; 1 \mathrm{H})$, double bond $=\mathrm{CH}_{2}\left(\delta 5.65, J_{\mathrm{BX}}=\right.$ 17.4 and $\delta 5.15, J_{\mathrm{AX}}=11.0, J_{\mathrm{AB}}=1.3 \mathrm{~Hz} ; \mathrm{AB}$ part of $\mathrm{ABX} ; 2 \mathrm{H})$, benzyl methylene $-\mathrm{CH}_{2}-$ $(\delta 4.5, \mathrm{~s} ; 2 \mathrm{H})$, oxyethylene $\left(\mathrm{OCH}_{2} \mathrm{CH}_{2}\right)_{n}$ $(\delta 3.64, \mathrm{~s}, 4 \bar{n} \mathrm{H})$, tert-butyl $(\delta 1.2 ; \mathrm{s} ; 9 \mathrm{H})$.

PEO-MA: double bond $=\mathrm{CH}_{2}(\delta 5.35$ and $\delta 6.1 ; \mathrm{m} ; 2 \mathrm{H})$, oxyethylene $\left(-\mathrm{OCH}_{2} \mathrm{CH}_{2}\right)_{n}$ $(\delta 4.3$ and $\delta 3.64 ; \mathrm{t}$ and $\mathrm{s} ; 4 \bar{n} \mathrm{H}), \alpha$-methyl $(\delta 1.95 ;$ br s; $3 \mathrm{H})$, tert-butyl $(\delta 1.2 ; \mathrm{s} ; 9 \mathrm{H})$.

The number-average degree of polymerization, $\bar{n}$, was estimated from the ratio of the peak area of oxyethylene to that of tert-butyl protons.

Typical NMR spectra of the styrenemacromonomer copolymerization products are given in Figures 1 and 2. The mole fraction of styrene, $x$, was calculated from the peak area of phenyl protons relative to that of other appropriate protons as follows.

\section{PEO-VB:}

$$
\frac{\text { area of phenyl protons at } \delta 6.3-7.2}{\text { area of } \mathrm{OCH}_{2} \mathrm{CH}_{2} \mathrm{O} \text { protons at } \delta 3.6}=\frac{5 x+4(1-x)}{4 \bar{n}(1-x)}
$$

(a)

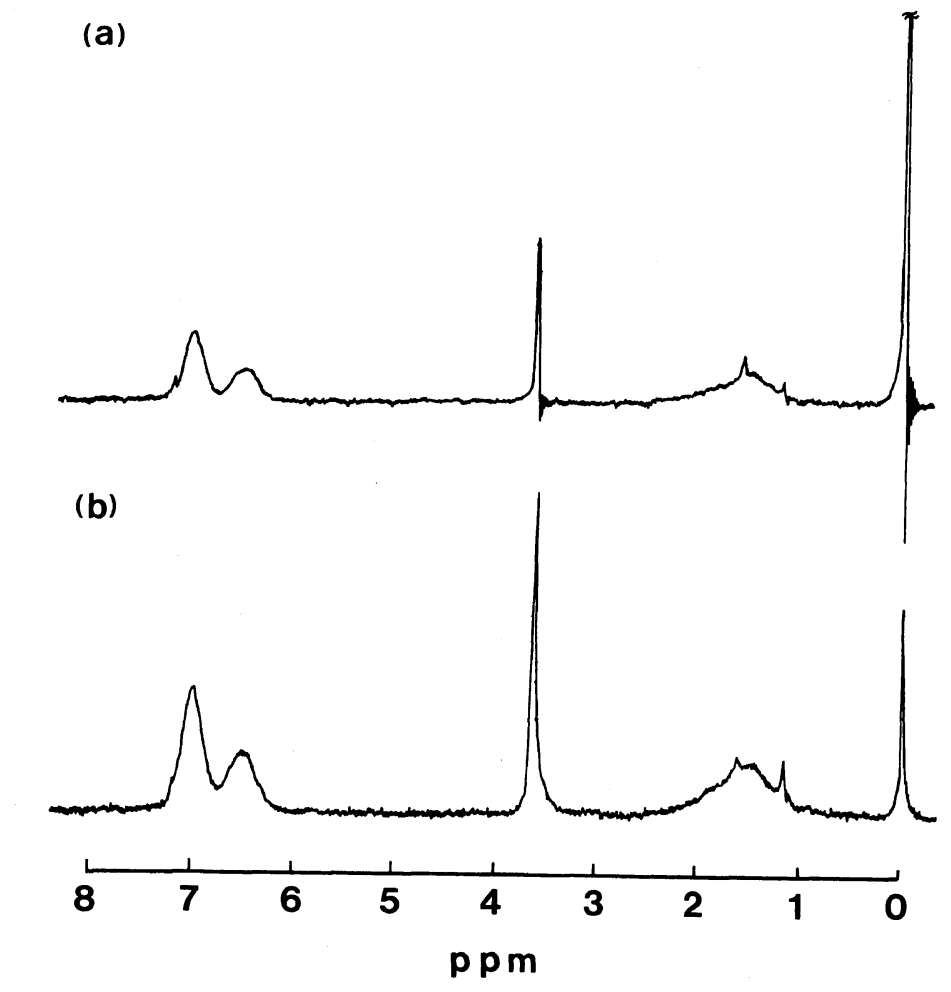

Figure 1. ${ }^{1} \mathrm{H}$ NMR spectra of the copolymerization products from styrene with (a) PEO-VB-29, $x=$ 0.987 , and (b) PEO-MA-25, $x=0.977(60 \mathrm{MHz})$. 


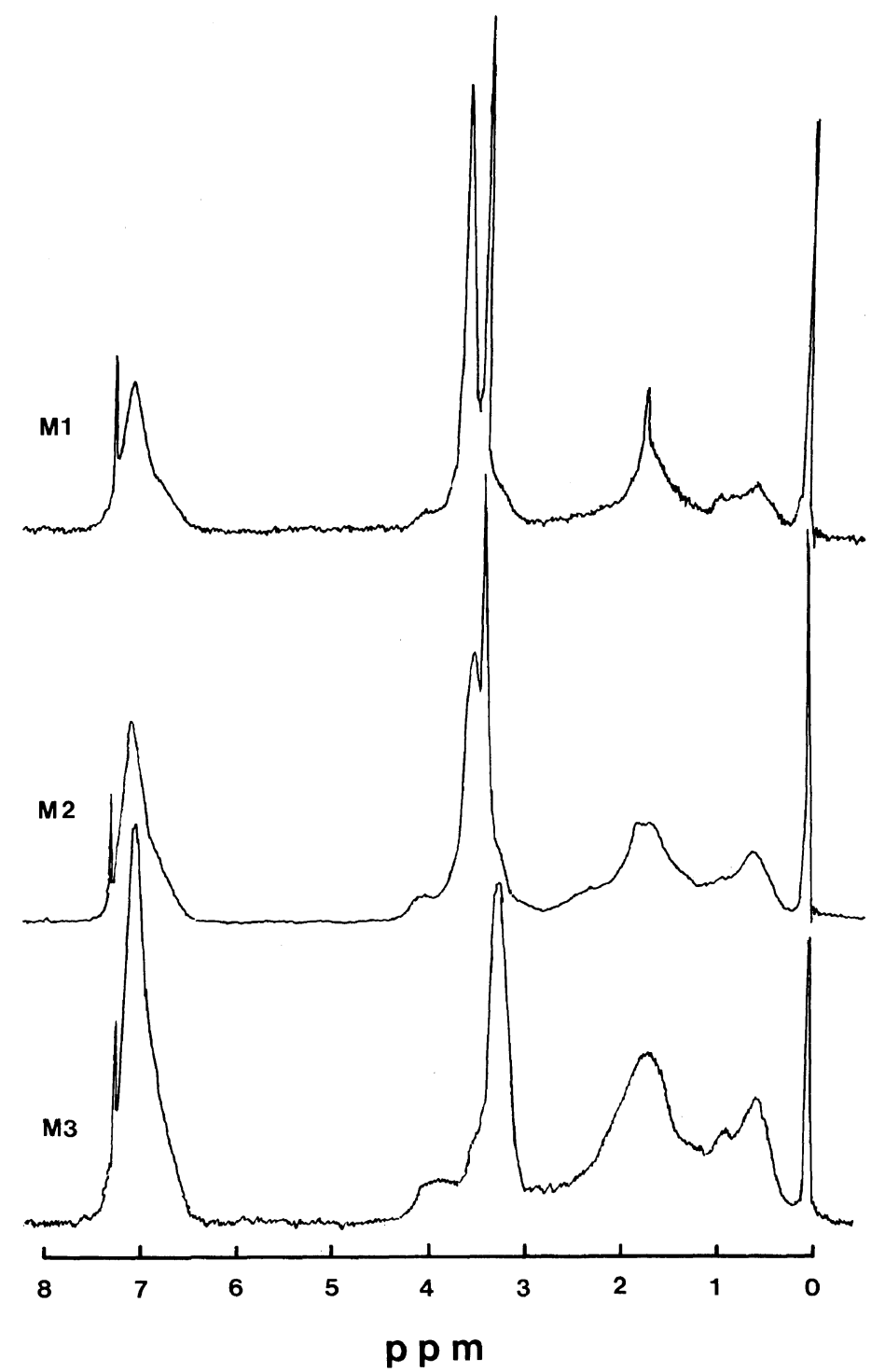

Figure 2. ${ }^{1} \mathrm{H}$ NMR spectra of the copolymers of styrene $\left(\mathrm{M}_{1}\right)$ with oligo(ethylene glycol) methyl ether methacrylates $\left(M_{2}\right)$. Feed molar ratio $\left[M_{1}\right] /\left[M_{2}\right]=60 / 40$.

\section{PEO-MA:}

$$
\frac{\text { area of phenyl protons at } \delta 7.2-6.3}{\text { area of all the other protons at } \delta 4.3-0.5}=\frac{5 x}{3 x+(4 \bar{n}+8)(1-x)}
$$

M1, M2, and M3:

$$
\frac{\text { area of phenyl protons at } \delta 7.0}{\text { area of all the other protons at } \delta 4.3-0.3}=\frac{5 x}{3 x+(4 n+8)(1-x)}
$$


( a )

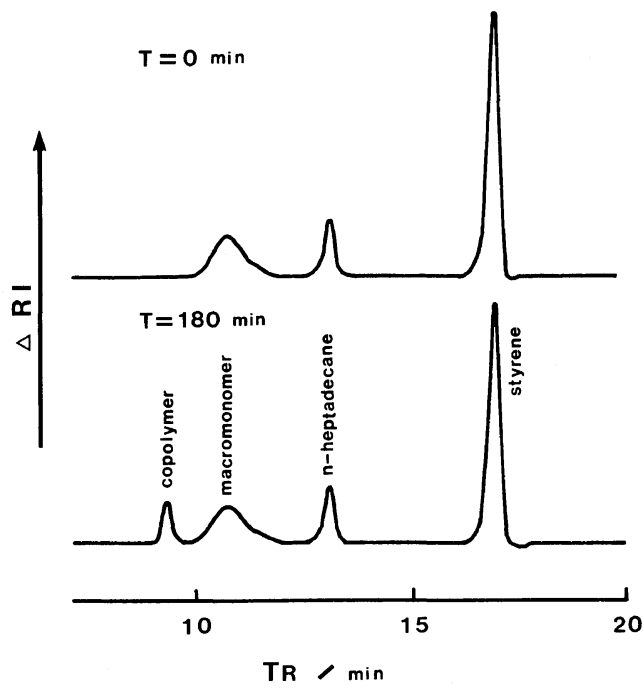

(b)

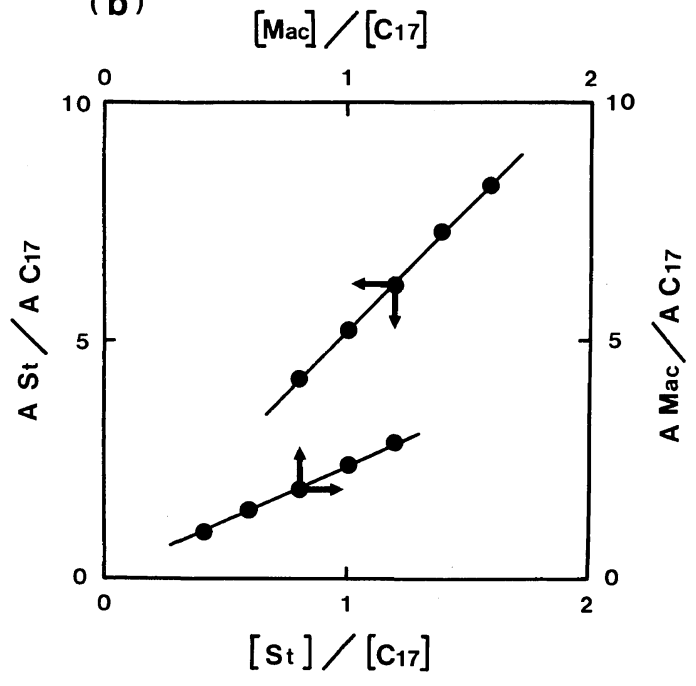

Figure 3. (a) GPC trace of the copolymerization of styrene and PEO-VB-29, and (b) calibration for peak areas, A, relative to heptadecane as the internal standard.

Table I. Characterization of PEO macromonomers

\begin{tabular}{|c|c|c|c|c|c|}
\hline \multirow{2}{*}{ Macromonomer } & \multirow{2}{*}{$\begin{array}{c}\mathrm{VPO} \\
\bar{n}\end{array}$} & \multirow{2}{*}{$\begin{array}{c}\mathrm{NMR}^{\mathrm{a}} \\
\bar{n}\end{array}$} & \multicolumn{2}{|c|}{ GPC } & \multirow{2}{*}{$\begin{array}{c}\mathrm{UV} \\
\bar{n}\end{array}$} \\
\hline & & & $\bar{n}$ & $M_{w} / M_{n}$ & \\
\hline PEO-VB-13 & 13.1 & 14.3 & $14.3^{\mathrm{b}}$ & 1.07 & 14.3 \\
\hline PEO-VB-29 & 28.6 & 31.5 & $30.9^{\mathrm{b}}$ & 1.09 & 33.4 \\
\hline PEO-VB-33 & 32.7 & 26.1 & $31.8^{\mathrm{c}}$ & 1.05 & 29.3 \\
\hline PEO-VB-34 & 33.9 & 31.7 & $35.0^{\mathrm{b}}$ & 1.11 & 37.5 \\
\hline PEO-VB-51 & 51.1 & 39.7 & $45.2^{\mathrm{c}}$ & 1.05 & 48.2 \\
\hline PEO-VB-77 & 76.6 & 69 & $74.3^{\mathrm{c}}$ & 1.04 & 87.5 \\
\hline PEO-VB-94 & 94.1 & 82 & $95.5^{\mathrm{c}}$ & 1.03 & 110 \\
\hline PEO-MA-25 & 25.4 & 24.5 & $25.0^{\mathrm{b}}$ & 1.12 & 28.4 \\
\hline PEO-MA-38 & 38.4 & 37.7 & $31.1^{\mathrm{b}}$ & 1.24 & 43.4 \\
\hline PEO-MA-62 & 62.2 & 59.3 & $52.6^{\mathrm{b}}$ & 1.09 & 70.9 \\
\hline
\end{tabular}

a Calculated from the ratio of the peak areas of oxyethylene to tert-butyl.

b Calculated by eq 1 with $M_{n}$ from polystyrene calibration.

c Using a calibration by PEO standard samples.

The choice of area of all other protons, instead of that of $\mathrm{OCH}_{2} \mathrm{CH}_{2} \mathrm{O}$ protons in the cases of methacrylates, eq 3 and 4 , was based on the possibility that some part, even if very little in the case of PEO-MA, of the ester $\alpha$-methylene protons, flanked by styrene units, should give rise to a peak at a considerably higher magnetic field, up to around $\delta 2.3$, as found in the case for styrene-methyl methacrylate and related copolymers. ${ }^{16}$

The composition of the copolymerization product from styrene and PEO-VB-29 was also checked by GPC, monitoring the decrease of the peak areas of the monomers relative to that of the internal standard, hexadecane, which had been weighed into the mixture. Figure 3 shows a typical run and the calibration of the peak area.

\section{RESULTS AND DISCUSSION}

\section{Macromonomers}

Table I summarizes the results of the characterization of $p$-vinylbenzyl- and methacrylateended poly(ethylene oxide) macromonomers, PEO-VB and PEO-MA, respectively. The number-average degrees of polymerization (DP), $\bar{n}$, agree satisfactorily with each other, irrespective of the method of determination. The DP distribution is sharp, with $M_{w} / M_{n}$ around 1.1. 

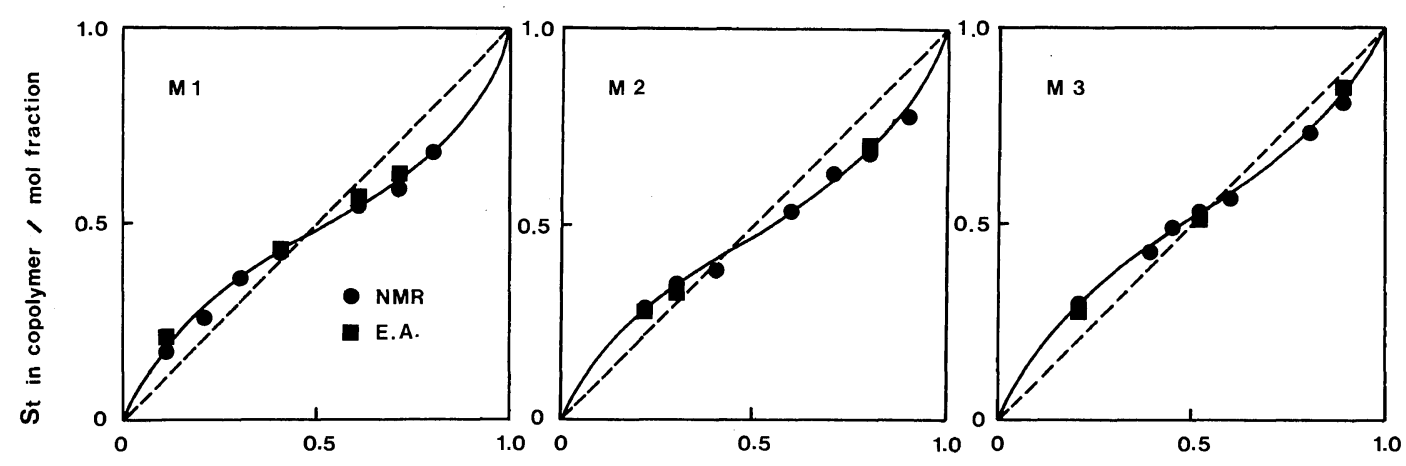

St in monomer / mol fraction

Figure 4. Composition curves for copolymerizations of styrene with oligo(ethylene glycol) methyl ether methacrylates. Total monomer $2 \mathrm{~g}$, THF $10 \mathrm{ml}$, AIBN $0.06 \mathrm{mmol}, 4-5 \mathrm{~h}, 10-20 \% \mathrm{conv}$. Analyses by (๑) ${ }^{1} \mathrm{H}$ NMR and ( $\left.\boldsymbol{\square}\right)$ elemental analysis for $\mathrm{C}$.

${ }^{1} \mathrm{H}$ NMR spectra of the macromonomers were reasonably consistent with the structure in both the chemical shifts and relative intensities of the respective peaks. UV spectra also confirmed the presence of $p$-vinylbenzyl and methacrylate end-groups at $\lambda_{\max }=252$ and $209 \mathrm{~nm}$, respectively, just as those reported for the corresponding polytetrahydrofuran macromonomers. ${ }^{13,14}$ Estimation of $\bar{n}$, using the reported $\varepsilon_{\max }$ assuming one terminal double bond per chain, was reasonably satisfactory for PEO-VB with $\bar{n}$ less than about 50. However, some discrepancy, as much as $15 \%$ higher in $\bar{n}$ compared to that determined by VPO, was apparent for the other macromonomers. The reason for this may possibly be due to either error involved in the determination or incomplete introduction of the double bond. Should the latter apply, the purity of the double bond in each macromonomer is in the range of 85 to $100 \%$, as calculated by $\bar{n}(\mathrm{VPO}) / \bar{n}(\mathrm{UV})$. Even so, it may be reasonable to conclude that the following discussions are still valid. The number-average DP of the macromonomer was taken throughout this paper to be that determined by VPO.

Determination of Copolymerization Parameters Figure 4 shows the copolymer-monomer
Table II. Monomer reactivity ratios for copolymerizations of styrene $\left(M_{1}\right)$ with oligo(ethylene glycol)methyl ether methacrylate $\left(\mathrm{M}_{2}\right)$

\begin{tabular}{ccc}
\hline $\mathrm{M}_{2}$ & $r_{1}$ & $r_{2}$ \\
\hline M1 & $0.37 \pm 0.13$ & $0.43 \pm 0.05$ \\
M2 & $0.38 \pm 0.08$ & $0.52 \pm 0.09$ \\
M3 & $0.49 \pm 0.09$ & $0.44 \pm 0.08$ \\
\hline
\end{tabular}

composition curves for copolymerizations of styrene $\left(M_{1}\right)$ with the low molecular weight homologs of PEO-MA, i.e., M1, M2, and M3. The monomer reactivity ratios were estimated by the method of Kelen and Tüdös, ${ }^{17}$ with the results given in Table II. No difficulty or abnormality was found in the determination of the $r_{1}$ and $r_{2}$ values. The copolymers obtained are clearly random in sequence distribution since the ${ }^{1} \mathrm{H}$ NMR spectra (Figure 3 ) showed a single phenyl peak together with relatively upfielded, complicated peaks due to ester $\alpha$ methylene and $\alpha$-methyl protons, as in the case of related copolymers. ${ }^{16}$

In the case of the conventional monomer $\left(\mathrm{M}_{1}\right)$ and macromonomer $\left(\mathrm{M}_{2}\right)$, difficulty arises from the necessity that the ratio of $M_{1}$ to $M_{2}$ is usually taken around unity, say from $1 / 3$ to $3 / 1$, on a weight basis and hence in a very high range on a molar basis for both analytical and preparative purposes. The molar 
composition of the copolymer should then be very insensitive to the value of $r_{2}$, since the conventional Mayo-Lewis copolymerization equation,

$$
\frac{\mathrm{d}\left[\mathrm{M}_{1}\right]}{\mathrm{d}\left[\mathrm{M}_{2}\right]}=\frac{1+r_{1}\left[\mathrm{M}_{1}\right] /\left[\mathrm{M}_{2}\right]}{1+r_{2}\left[\mathrm{M}_{2}\right] /\left[\mathrm{M}_{1}\right]}
$$

reduces to, in the limiting condition of $\left[\mathrm{M}_{1}\right] /$ $\left[\mathrm{M}_{2}\right] \gg 1,{ }^{18,19}$

$$
\frac{\mathrm{d}\left[\mathrm{M}_{1}\right]}{\mathrm{d}\left[\mathrm{M}_{2}\right]}=r_{1} \frac{\left[\mathrm{M}_{1}\right]}{\left[\mathrm{M}_{2}\right]}
$$

This is equivalent to saying that we are effectively dealing with only two of four conventional propagation reactions:

$$
\begin{aligned}
& \sim \mathrm{M}_{1} \cdot+\mathrm{M}_{1} \stackrel{k_{11}}{\longrightarrow} \sim \mathrm{M}_{1} . \\
& \sim \mathrm{M}_{1} \cdot+\mathrm{M}_{2} \stackrel{k_{12}}{\longrightarrow} \sim \mathrm{M}_{2} .
\end{aligned}
$$

with

$$
r_{1}=k_{11} / k_{12}
$$

together with the implicit condition of the stationary state that the other crosspropagation

$$
\sim \mathrm{M}_{2} \cdot+\mathrm{M}_{1} \stackrel{k_{21}}{\longrightarrow} \sim \mathrm{M}_{1} .
$$

should occur with a rate equal to that of the reaction 8 , so that

$$
k_{12}\left[\sim \mathrm{M}_{1} \cdot\right]\left[\mathrm{M}_{2}\right]=k_{21}\left[\sim \mathrm{M}_{2} \cdot\right]\left[\mathrm{M}_{1}\right]
$$

Hence, with the condition $\left[\mathrm{M}_{1}\right] /\left[\mathrm{M}_{2}\right] \gg 1$, we may neglect the contribution of polymer radical $\sim \mathrm{M}_{2} \cdot$ on the composition equation.

Therefore, the $r_{1}$ thus determined should approximate most simply the relative reactivities of the monomers $\mathbf{M}_{1}$ and $\mathbf{M}_{2}$ in competition reactions with the $M_{1}$-ended radical which should also approximate a homopoly$M_{1}\left(M_{1}\right.$-consecutive $)$ radical. We thus need not consider any complication due to penul-

\begin{tabular}{|c|c|c|c|c|c|c|c|}
\hline \multirow{3}{*}{ Run } & \multicolumn{3}{|c|}{ Feed } & \multirow{3}{*}{$\frac{\text { Time }}{\mathrm{h}}$} & \multirow{3}{*}{$\frac{\text { Yield }^{\mathrm{b}}}{w \mathrm{t}^{\circ} \%}$} & \multirow{2}{*}{\multicolumn{2}{|c|}{$\begin{array}{c}\text { Polymer } \\
\mathrm{M}_{1} / \mathrm{mol}^{\circ} / \mathrm{o}\end{array}$}} \\
\hline & $\mathbf{M}_{1}$ & $\mathbf{M}_{2}$ & $\mathbf{M}_{1}$ & & & & \\
\hline & g & $\mathrm{g}$ & $\mathrm{mol}^{\circ} \%$ & & & NMR & GPC \\
\hline 1 & 0.803 & 1.202 & 90.3 & 3.0 & 6.1 & 92.3 & 92.4 \\
\hline 2 & 1.001 & 1.000 & 93.3 & 3.0 & 7.7 & 94.7 & 94.4 \\
\hline 3 & 1.201 & 0.801 & 95.4 & 3.0 & 5.8 & 96.4 & 96.5 \\
\hline 4 & 1.396 & 0.604 & 97.0 & 3.0 & 6.4 & 97.8 & - \\
\hline 5 & 1.605 & 0.407 & 98.2 & 3.2 & 8.1 & 98.7 & 98.6 \\
\hline
\end{tabular}
timate effects or depropagation, which might possibly occur in propagation reactions involving a more crowded radical as suspected in the case of increased composition of the mac-
Table III. Copolymerization of styrene $\left(\mathrm{M}_{1}\right)$ and PEO-VB-29 $\left(\mathrm{M}_{2}\right)^{\mathrm{a}}$

a AIBN $c a .20 \mathrm{mg}$, THF ca. $4.7 \mathrm{~g}$, heptadecane $c a .1 .0 \mathrm{~g}$, polymerization at $60^{\circ} \mathrm{C}$.

b Polymers recovered by precipitation into petroleum ether, followed by repeated washing with water.

romonomer $\left(\mathrm{M}_{2}\right)$. It should also be noted that the $r_{1}$ values obtained by copolymerization of a common monomer $\left(\mathrm{M}_{1}\right)$, styrene in the present case, with various monomers $\left(\mathrm{M}_{2}\right)$ can be a measure of the relative reactivities of the monomers $\mathbf{M}_{2}$ by taking their reciprocals, $1 / r_{1}=k_{12} / k_{11}$.

Now we turn to the results of the application of eq 5 and 6 to the composition data of copolymerization of styrene $\left(\mathrm{M}_{1}\right)$ with PEOVB-29, as an example. As shown in Table III, the copolymer compositions were necessarily very rich in styrene on a molar basis but could be satisfactorily determined by ${ }^{1} \mathrm{H}$ NMR and GPC, although the latter method was somewhat less sensitive to the change in composition at such a low conversion. Application of the method of Kelen and Tüdös, ${ }^{17}$ which is based on eq 5 , gave $r_{1}=1.36 \pm 0.08$ and $r_{2}=$ $1.47 \pm 1.19$, with error of $95 \%$ confidence limit. As expected, the value of $r_{2}$ involves too much error to be reliable. On the other hand, $r_{1}$ value is quite significant and in good agreement with that obtained by application of eq 6 , that is $r_{1}=1.33 \pm 0.07$. These results clearly justify the application of the approximate equation 6 to the present copolymerization.

Tables IV and V summarize the composition data for the copolymerizations of styrene with 
Table IV. Copolymerization of styrene $\left(M_{1}\right)$ and PEO-VB $\left(\mathrm{M}_{2}\right)$

\begin{tabular}{|c|c|c|c|c|}
\hline \multirow{2}{*}{$\mathrm{M}_{2}$} & {$\left[\mathrm{M}_{1}\right] /\left[\mathrm{M}_{2}\right]$} & Time & Yield & $\mathrm{d}\left[\mathrm{M}_{1}\right] / \mathrm{d}\left[\mathrm{M}_{2}\right]$ \\
\hline & $\mathrm{mol} / \mathrm{mol}$ & $\mathrm{h}$ & $w t \%$ & $\mathrm{~mol} / \mathrm{mol}$ \\
\hline \multirow[t]{4}{*}{ PEO-VB-1 $3^{\mathrm{a}}$} & 21.7 & 3.2 & 11.7 & 19.8 \\
\hline & 12.5 & 3 & 10.2 & 13.0 \\
\hline & 7.8 & 3 & 8.9 & 8.1 \\
\hline & 4.5 & 3 & 7.5 & 4.7 \\
\hline \multirow[t]{5}{*}{ PEO-VB-33 } & 297 & 18 & 18.3 & 444 \\
\hline & 192 & 18 & 18.4 & 295 \\
\hline & 142 & 18 & 16.6 & 213 \\
\hline & 109 & 18 & 17.2 & 166 \\
\hline & 89 & 18 & 12.8 & 142 \\
\hline \multirow[t]{4}{*}{ PEO-VB- $34^{\mathrm{b}}$} & 148 & 24 & 18.0 & 202 \\
\hline & 91 & 24 & 19.0 & 123 \\
\hline & 64 & 24 & 18.5 & 82 \\
\hline & 38 & 24 & 7.0 & 54 \\
\hline \multirow[t]{5}{*}{ PEO-VB- $51^{b}$} & 446 & 19 & 21.1 & 757 \\
\hline & 289 & 18 & 18.9 & 487 \\
\hline & 212 & 19 & 14.0 & 355 \\
\hline & 165 & 18 & 16.1 & 281 \\
\hline & 133 & 18 & 11.4 & 237 \\
\hline \multirow[t]{4}{*}{ PEO-VB- $77^{b}$} & 651 & 18 & 16.6 & 1110 \\
\hline & 308 & 18 & 14.3 & 551 \\
\hline & 239 & 18 & 16.5 & 434 \\
\hline & 192 & 18 & 6.3 & 338 \\
\hline \multirow[t]{5}{*}{ PEO-VB-94 } & 1630 & 18 & 19.5 & 3010 \\
\hline & 785 & 18 & 18.7 & 1390 \\
\hline & 781 & 18 & 17.7 & 1480 \\
\hline & 510 & 18 & 19.1 & 839 \\
\hline & 374 & 18 & 14.4 & 670 \\
\hline
\end{tabular}

a AIBN $1 \mathrm{~mol} \%$ to total monomer, total monomer $30 \mathrm{wt} \%$ in THF. Polymers were recovered by precipitation into petroleum ether, followed by repeated washing with water.

b AIBN $1 \mathrm{~mol} \%$ to total monomer, total monomer $2 \mathrm{~g}$ in $20 \mathrm{ml}$ THF. Polymers were recovered by precipitation into methanol.

PEO-VB and -MA, respectively. Tables VI and VII summarize the corresponding monomer reactivity ratios, $r_{1}$, obtained by application of eq 6 , together with those for the low molecular weight reference monomers reported in the literature. ${ }^{20-23}$ It should be noted here that the molecular weights of the copolymerization products, as estimated by GPC calibrated with standard polystyrenes, were in the order of
Table V. Copolymerization of styrene $\left(M_{1}\right)$ and PEO-MA $\left(\mathrm{M}_{2}\right)^{\mathrm{a}}$

\begin{tabular}{|c|c|c|c|c|}
\hline \multirow{2}{*}{$\mathrm{M}_{2}$} & {$\left[\mathrm{M}_{1}\right] /\left[\mathrm{M}_{2}\right]$} & Time & Yield & $\mathrm{d}\left[\mathrm{M}_{1}\right] / \mathrm{d}\left[\mathrm{M}_{2}\right]$ \\
\hline & $\mathrm{mol} / \mathrm{mol}$ & $\mathrm{h}$ & $w t \%$ & $\mathrm{~mol} / \mathrm{mol}$ \\
\hline \multirow[t]{5}{*}{ PEO-MA-25 } & 102 & 44 & 18 & 90 \\
\hline & 59 & 13 & 6 & 57 \\
\hline & 46 & 13 & 3 & 43 \\
\hline & 40 & 34 & 10 & 42 \\
\hline & 32 & 59 & 11 & 38 \\
\hline \multirow[t]{5}{*}{ PEO-MA-38 } & 155 & 96 & 21 & 136 \\
\hline & 98 & 109 & 12 & 105 \\
\hline & 82 & 50 & 5 & 90 \\
\hline & 69 & 96 & 16 & 73 \\
\hline & 58 & 50 & 3 & 65 \\
\hline \multirow[t]{7}{*}{ PEO-MA-62 } & 525 & 45 & 10 & 454 \\
\hline & 249 & 96 & 3 & 302 \\
\hline & 226 & 110 & 12 & 262 \\
\hline & 155 & 144 & 4 & 184 \\
\hline & 124 & 110 & 1.3 & 158 \\
\hline & 111 & 164 & 1.4 & 151 \\
\hline & 93 & 112 & 0.1 & 113 \\
\hline
\end{tabular}

a AIBN $1 \mathrm{~mol} \%$ to total monomer, total monomer $2 \mathrm{~g}$ in $20 \mathrm{ml}$ THF. Polymers were recovered by precipitation into methanol, followed by centrifuging.

$2 \times 10^{4}$, suggesting the possibility of the occurrence of some homopolystyrenes in the products, which should be, however, included for estimation of $r_{1}$ as long as this value means the reactivity ratio as defined by eq 9 with reactions 7 and 8 . Finally, it is to be mentioned that the data in Tables IV and $\mathrm{V}$ were also analyzed in terms of the integrated form of eq $6,{ }^{24}$ to give almost the same values of $r_{1}$ as those in Tables VI and VII, indicating the negligible effect of the conversions, up to about $30 \%$, on compositions not so far from the azeotrope.

\section{Effects of Molecular Weight on Macromono- mer's Reactivity}

Since monomer $\mathbf{M}_{1}$ is styrene throughout this work, the reciprocal of $r_{1}$ is a measure of the relative reactivity of the monomer $\mathbf{M}_{2}$, compared to styrene as a reference, toward a common polystyrene radical. Inspection of 
Table VI. Monomer reactivity ratio of styrene $\left(M_{1}\right)$ in copolymerization with PEO-VB macromonomers $\left(\mathrm{M}_{2}\right)$

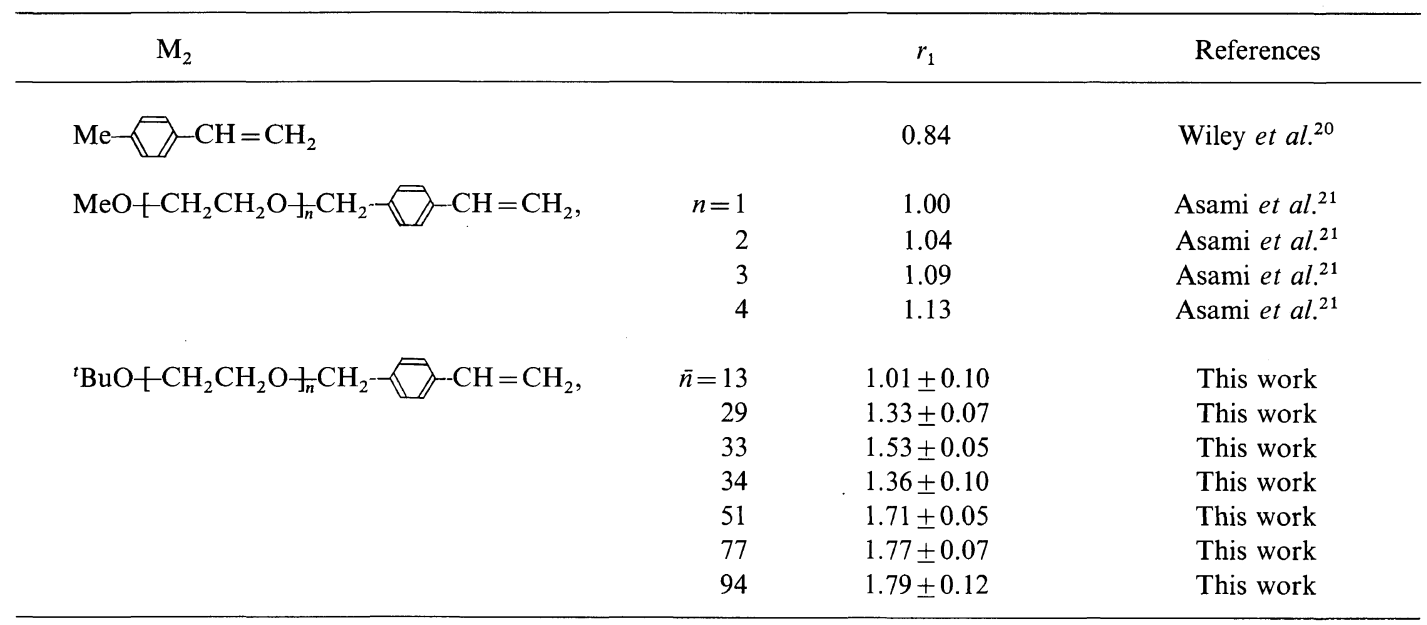

Table VII. Monomer reactivity ratio of styrene $\left(\mathrm{M}_{1}\right)$ in copolymerization with PEO-MA macromonomers $\left(\mathrm{M}_{2}\right)$

\begin{tabular}{|c|c|c|c|}
\hline $\mathrm{M}_{2}$ & & $r_{1}$ & References \\
\hline \multirow{6}{*}{ 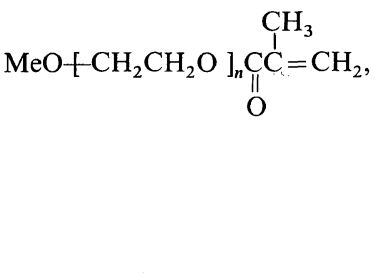 } & & & \\
\hline & $n=0$ & $0.52 \pm 0.026$ & Lewis et $a .^{22}$ \\
\hline & 1 & $0.50 \pm 0.01$ & Yokota et al. . $^{23}$ \\
\hline & 1 & $0.37 \pm 0.13$ & This work \\
\hline & 2 & $0.38 \pm 0.08$ & This work \\
\hline & 3 & $0.49 \pm 0.09$ & This work \\
\hline \multirow{4}{*}{${ }^{\mathrm{BuO}}\left[\mathrm{CH}_{2} \mathrm{CH}_{2} \mathrm{O}+_{n} \stackrel{\mathrm{C}}{\mathrm{C}} \underset{\mathrm{O}}{\mathrm{C}}=\mathrm{CH}_{2}\right.}$, & & & \\
\hline & $\bar{n}=25$ & $0.94 \pm 0.13$ & This work \\
\hline & 35 & $1.02 \pm 0.12$ & This work \\
\hline & 62 & $1.18 \pm 0.11$ & This work \\
\hline
\end{tabular}

Tables VI and VII shows first that the reactivities of the low molecular weight homologs with $n \leqq 4$ do not change significantly. This is expected since change in $\mathrm{R}$ or $n$ in $\mathbf{M}_{2}$ would little influence the reactivity of the double bond, located at a considerably separated possition, both inductively and sterically. This is true even with $p$-methylstyrene and methyl methacrylate as $\mathbf{M}_{2}$. Any solvent effect, found significantly large in the copolymerization of 2-hydroxyethyl methacrylate, ${ }^{6}$ cannot be expected here for the present monomers with little possibility of hydrogen-bonding aggregation.

Secondly, most important in Tables VI and VII is that the relative reactivities of the macromonomers, both PEO-VB and PEO-MA, decrease with increasing degree of polymerization, as is clear in the plots in Figure 5. This apparently indicates the effect of the PEO polymer chain to reduce the reactivity of the terminal bond. This cannot be ascribed to the inductive or steric effect in the sence of conventional organic chemistry, but to certain 


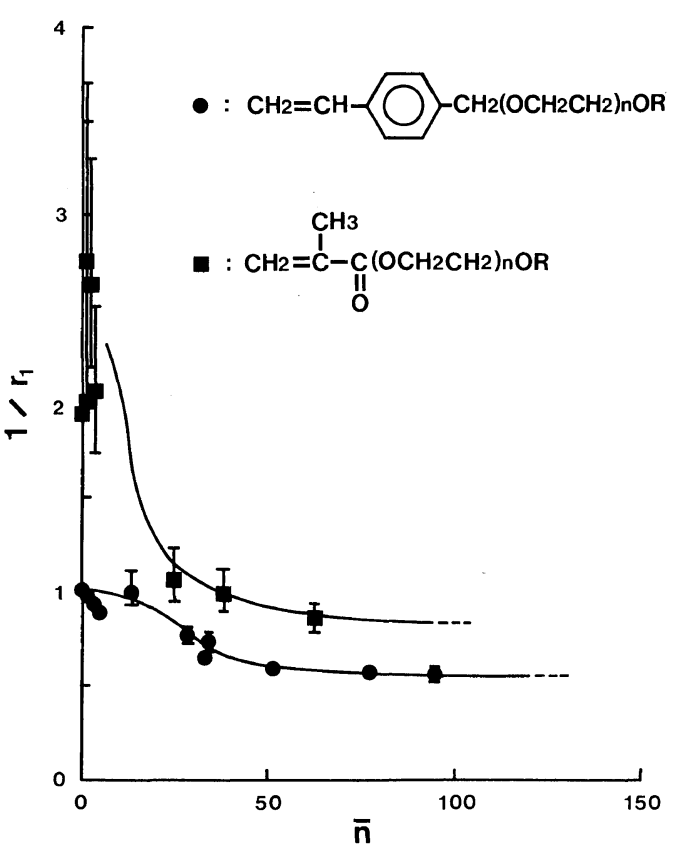

Figure 5. Relative reactivities of macromonomers as a function of the degree of polymerization.

polymer effect(s) arising from the solution propertis of the macromolecular reactants, except that a somewhat greater reduction was observed in the reactivity of PEO-MA as compared to PEO-VB. The last fact remains most difficult to explain at present.

Since the reaction here is a radical-monomer propagation with a rate constant of the order of $10^{2} 1 \mathrm{~mol}^{-1} \mathrm{~s}^{-1}$ and an activation energy of $5-10 \mathrm{kcal} \mathrm{mol}^{-1}$, it is most reasonable to say, at least for a reaction in a relatively dilute solution to a relatively low conversion, that it is chemically controlled, but not physically (diffusion) controlled as in the case of a radical-radical termination reaction. ${ }^{25}$ One of the most probable explanations for the observed polymer effect is that of the kinetic excluded volume effect as proposed by Morawetz et $a l .^{26}$ This concept is based on the polymer solution theory that polymer segments cannot interpenetrate freely, thus decreasing the probability of an encounter of the reactive groups belonging to different polymer chains. Depending on the model for calculation, the theoretical relations derived are that the second-order rate constants should decrease in proportion to -0.15 (Morawetz et $a l^{26}$ ), -0.10 and -0.07 (Okamoto et $a .^{27}$ ) powers of the DP for reactions between the end-groups belonging to different polymer chains which are identical except for the reactive end-groups. Few experimental studies, however, have clearly demonstrated this prediction. Okamoto et al. recently examined the reaction of polystyrene living anions with chloro-ended polystyrenes ${ }^{28}$ and that of amino-ended with chlorosulfonyl-ended poly(ethylene oxides) ${ }^{27}$ over a wide range of DP, up to 400 and 20000 , respectively. The results, however, revealed very little dependence on DP, and they pointed out that the "kinetic excluded volume effect," if any, should be actually even less than those predicted above by introducing a theory of higher-order approximation.

The situation is considerably different from the above in the present copolymerization in that the polymer reactants are different in nature, one being a polystyrene radical and the other a PEO macromonomer. Since these polymers are incompatible ${ }^{29}$ or interact repulsively, they should interpenetrate in solution with greater difficulty than in the case of the same polymers, thus allowing a corresponding decrease in the apparent reactivity as observed. Thus, a theory including an interaction, which should be generally repulsive, between different polymers appears to provide a basis for the observed effect. In a solution theory for a polymer-polymer-solvent ternary system, developed earlier by Scott, the critical polymerpolymer interaction parameter, above which the polymers segregate as incompatible, should decrease almost inversely with increasing DP. ${ }^{30,31}$ Asami et al..$^{32}$ reported that the reactivities of $p$-vinylbenzyl- or $p$-vinylphenoxy-ended polytetrahydrofuran macromonomers in homopolymerizations are very similar to those of their low molecular weight 
models, a result which may be expected from the above concept since the polymers involved are of the same nature here.

An alternative explanation, which should be considered, is a decrease in reactivity as a result of some conformational or aggregation effect, possibly peculiar to PEO chains, since they became less soluble in THF with increasing DP and crystallized out of the solvent at room temperature around $20^{\circ} \mathrm{C}$, though the copolymerization systems remained apparently homogeneous at $60^{\circ} \mathrm{C}$. It is difficult, however, to get a clear picture of this situation. As stated in the introduction, the macromonomer's reactivity has been reported similar or reduced, compared to the reference monomers, depending on the nature of the macromonomer-comonomer pairs. The concepts of the interaction between different polymers and/or the difference in the solution behavior may be useful for explaining the results in each case.

In conclusion, the reactivities of PEO-VB and -MA macromonomers in copolymerizations with styrene were found to decrease significantly with increasing DP. A repulsive interaction between different polymer chains, rather than the kinetic excluded volume effect in its original form, and/or some difference in solution behavior were pointed out as possible factors influencing the macromonomer's reactivity, which is important for the design of composition and structure of graft copolymers.

Acknowledgements. The authors express their appreciation to Dr. Katsukiyo Ito, Government Industrial Research Institute, Nagoya, and Dr. Kazuyuki Horie, University of Tokyo, for their helpful comments on this work. Standard poly(ethylene oxides) were kindly supplied from Toyo Soda Mfg. Co., Ltd. This work was supported in part by a fund from Nippon Oil \& Fats Co., Ltd.

\section{REFERENCES}

1. Y. Yamashita, Kobunshi, 31, 988 (1982).

2. P. F. Rempp and E. Franta, Adv. Polym. Sci., 58, 1 (1984).

3. K. Ito, N. Usami, and Y. Yamashita, Macromolecules, 13, 216 (1980).

4. K. Ito, Y. Masuda, T. Shintani, T. Kitano, and Y. Yamashita, Polym. J., 15, 443 (1983).

5. S. Yamashita, K. Shibatani, K. Takakura, K. Imai, Kobunshi Ronbunshu, 39, 187 (1982).

6. K. Ito, K. Uchida, T. Kitano, E. Yamada, and T. Matsumoto, Polym. J., 17, 761 (1985).

7. G. O. Schulz and R. Milkovich, J. Polym. Sci., Polym. Chem. Ed., 22, 1633 (1984).

8. (a) R. Asami, M. Takaki, and T. Matsuse, Preprints, 1st SPSJ IPC, Kyoto, 1984, p 76; (b) M. Takaki, R. Asami, H. Hanahata, N. Sukenaga, and K. Sogawa, Polym. Prepr., Jpn., 30, 860 (1981).

9. J. P. Kennedy and M. Hiza, J. Polym. Sci., Polym. Chem. Ed., 21, 1033 (1983).

10. P. Masson, G. Beinert, E. Franta, and P. Rempp, Polym. Bull., 7, 17 (1982).

11. S. Itsuno, K. Yamazaki, K. Ito, E. Yamada, and T. Matsumoto, Polym. Prepr., Jpn., 33, 466 (1984).

12. K. Ito, Y. Hashizuka, and Y. Yamashita, Macromolecules, 10, 821 (1977).

13. R. Asami, M. Takaki, K. Kita, and E. Asakura, Polym. Bull., 2, 713 (1980).

14. M. Takaki, R. Asami, and T. Kuwabara, Polym. Bull., 7, 521 (1982).

15. S. Mori, Anal. Chem., 53, 1813 (1981).

16. For example, K. Ito, and Y. Yamashita, J. Polym. Sci., B, 3, 625 (1965); K. Ito, S. Iwase, K. Umehara, and Y. Yamashita, Kogyo Kagaku Zasshi, 70, 993 (1967).

17. T. Kelen and F. Tüdös, J. Macromol. Sci., Chem., A9, 1 (1975).

18. R. Milkovich and M. T. Chiang, US Patent 3,786,916 (1974).

19. J. Sierra-Vargas, E. Franta, and P. Rempp, Makromol. Chem., 182, 2603 (1981).

20. R. H. Wiley and B. Davis, J. Polym. Sci., 46, 423 (1960).

21. R. Asami and N. Nakajima, Polym. Prepr., Jpn., 24, 19 (1975).

22. F. M. Lewis, C. Walling, W. Cumming, E. Briggs, and F. R. Mayo, J. Am. Chem. Soc., 70, 1519 (1948).

23. K. Yokota, M. Kani, and Y. Ishii, J. Polym. Sci., A1, 6, 1325 (1968).

24. J. P. Kennedy and C. Y. Lo, Polym. Bull., 8, 63 (1982).

25. For example, (a) P. E. M. Allen and C. R. Patrick, "Kinetics and Mechanisms of Polymerization Reactions," Ellis Horwood Ltd., Sussex, 1974, Chapter 2; (b) K. Horie, Kobunshi, 32, 196 (1983).

26. H. Morawetz, J.-R. Cho, and P. J. Gans, Macro- 


\section{Reactivity of Poly(ethylene oxide) Macromonomers}

molecules, 6, 624 (1973).

27. A. Okamoto, K. Toyoshima, and I. Mita, Eur. Polym. J., 19, 341 (1983).

28. A. Okamoto, Y. Shimanuki, and I. Mita, Eur. Polym. J., 18, 545 (1982).

29. For example, T. Suzuki, Y. Murakami, T. Inui, and Y. Takegami, Polym. J., 13, 1027 (1981).
30. R. L. Scott, J. Chem. Phys., 17, 279 (1949).

31. S. Krause, in "Polymer Blends," Vol. 1, D. R. Paul and S. Newman, Ed., Academic Press, New York, N.Y., 1978, p. 15.

32. R. Asami, M. Takaki, K. Kyuda, and N. Sukunaga, Polym. J., 15, 261 (1983). 